\title{
Theoretical Model for Optical Spectrum and Structural Dynamics of $\mathrm{La}_{2-\mathrm{x}} \mathrm{Sr}_{\mathrm{x}} \mathrm{CuO}_{4}$
}

\author{
Sven Larsson ${ }^{1}$ \\ ${ }^{1}$ Department of Chemistry, Chalmers University of Technology, S-412 96 Göteborg, Sweden \\ Correspondence: Sven Larsson, Department of Chemistry, Chalmers University of Technology, S-412 96 \\ Göteborg, Sweden. E-mail: slarsson@chalmers.se
}

Received: May 13, 2014 Accepted: May 21, 2015 Online Published: June 18, 2015

doi:10.5539/apr.v7n4p14 URL: http://dx.doi.org/10.5539/apr.v7n4p14

\begin{abstract}
Superconductivity in cuprates cannot be fully understood using free-electron theory and the BCS model. Experiments during the last thirty years have shown that structural dynamics and localization cannot be ignored. The Marcus model is applied here for a theoretical assignment of the optical spectra. Hubbard-U absorption at $\approx 2 \mathrm{eV}$ is assigned as metal/metal (MM) charge transfer transition of type $2 \mathrm{Cu}(\mathrm{II})+\mathrm{h} v \rightarrow \mathrm{Cu}(\mathrm{I})+\mathrm{Cu}(\mathrm{III})$. The mid-infrared (MIR) sharp line at $0.35 \mathrm{eV}$ in $\mathrm{La}_{2} \mathrm{CuO}_{4}$ is interpreted as a vibrational transition with intensity borrowing from the crossing $\mathrm{Cu}(\mathrm{I})+\mathrm{Cu}(\mathrm{III})$ energy curve. Hole-doping decreases $\mathrm{U}$, since holes become available as acceptors. The relationship between the pseudogap and $\mathrm{U}$ in the doped cuprate is discussed. The absorption at $0.13 \mathrm{eV}$ and the activation energy at $0.035 \mathrm{eV}$, existing only after doping, are very likely due to vertical and adiabatic electron exchange of type $\mathrm{Cu}(\mathrm{III})+\mathrm{Cu}(\mathrm{II}) \rightarrow \mathrm{Cu}(\mathrm{II})+\mathrm{Cu}(\mathrm{III})$. The pseudogap involves two-electron states.
\end{abstract}

Keywords: Cuprates, superconductivity (SC), doping, vibronic states, Hubbard-U, MIR absorption, pseudogap, metal-metal charge transfer

\section{Introduction}

Impressive progress towards an understanding of superconductivity (SC) in cuprates has been made since the discovery in $\mathrm{La}_{2-\mathrm{x}} \mathrm{Sr}_{\mathrm{x}} \mathrm{CuO}_{4}$ in 1986 (Bednorz \& Müller, 1986). Experimental methods which directly probe the electronic and vibrational wave functions have been advanced (Herr et al., 1987; Preyer et al., 1989; Tokura et al., 1990; Uchida et al., 1991; Thomas, Rapkine, Cooper, Cheong, \& Cooper, 1991; Thomas et al., 1992; Perkins et al., 1993). Theoretical progress has been hampered by the desire to keep as much as possible from the Bardeen-Cooper-Schrieffer (BCS) picture, including the free-electron model of electrons. However, vibrational and electronic coordinates interact in a localized way. Delocalization results only "after" this interaction is included, if $x>0.05$ (Larsson, 1989).

Various spectroscopies, such as absorption spectroscopies, photo-induced charge transfer in the infrared and visible region demonstrate localization very clearly. The pure cuprate $\mathrm{La}_{2} \mathrm{CuO}_{4}(\mathrm{x}=0)$ is a Mott insulator and has very little absorption in the low-energy region below $1.7 \mathrm{eV}$, except a mysterious weak absorption at $0.3-0.5 \mathrm{eV}$ in the mid-infrared (MIR) region (Perkins et al., 1993). Strong and wide absorption starts at about $1.8 \mathrm{eV}$, commonly accepted as due to charge transfer, but there is no agreement on which type of charge transfer. However, this is of crucial importance for the understanding of SC.

The present author suggested already in 1989 (Larsson, 1989) that the Marcus model (Marcus, 1964; Hush, 1967; Reimers \& Hush, 2004; Kestner, Logan, \& Jortner, 1974; Jortner, 1976; Jortner \& Bixon, 1969; Brunschwig, Logan, Newton, \& Sutin, 1980; Larsson, 1981; Klimkāns \& Larsson, 2000), if applied to electron pairs, would be a useful model for SC in cuprates. In the Born-Oppenheimer approximation the free energy of the "spin-coupled" $\mathrm{Cu}(\mathrm{II}) / \mathrm{Cu}$ (II) state and the "charged" $\mathrm{Cu}(\mathrm{I}) / \mathrm{Cu}(\mathrm{III})$ state form a common potential energy surface, consisting of two interacting paraboloids with partly common vibrational states (Larsson, 1989).

Conductivity in doped cuprates and $\mathrm{CuO}$ (Heikes \& Johnston, 1957; Larsson, 1986) results from oxidative doping, making the following activated electron transfer reaction possible:

$$
\mathrm{Cu}(\mathrm{II})+\mathrm{Cu}(\mathrm{III}) \leftrightarrow \mathrm{Cu}(\mathrm{III})+\mathrm{Cu}(\mathrm{II})
$$


A part of the optical conductivity (Falck, Levy, Kastner, \& Birgeneau, 1993; Kastner, Birgeneau, Shirane, \& Endoh, 1998; Basov, \& Timusk, 2005) is due to vertical excitation in the Marcus paraboloids, corresponding to $\mathrm{Cu}(\mathrm{II}) / \mathrm{Cu}(\mathrm{III})$ hole exchange.

The appearance of localized $\mathrm{Cu}(\mathrm{III})$ sites in the $\mathrm{CuO}_{2}$ plane after doping was confirmed with the discovery of stripes (Tranquada, Sternlieb, Axe, Nakamura, \& Uchida,1995). In $\mathrm{La}_{2-\mathrm{x}} \mathrm{Sr}_{\mathrm{x}} \mathrm{CuO}_{4} \mathrm{SC}$ arises for $\mathrm{x}>0.05$ due to interaction between the antiferromagnetic ground state and the charged state (Klimkans \& Larsson, 2001; Larsson, 2010, 2012). The parallel $\mathrm{CuO}_{2}$ planes are known to be responsible for SC. Spectra of different cuprates are similar, with a strong absorption covering a large part of the visible spectrum, including the ligand field transitions (Mott, 1968). We will mainly discuss optical conductivity spectra, and reflectivity spectra, particularly in the MIR region (Uchida et al., 1991; Falck, Levy, Kastner, \& Birgeneau, 1993; Kastner, Birgeneau, Shirane, \& Endoh, 1998; Basov, \& Timusk, 2005).

Since $\mathrm{Cu}(\mathrm{III})$ sites require less energy to accept electrons than $\mathrm{Cu}(\mathrm{II})$ sites, intensity builds up in the region below $1.2 \mathrm{eV}$ after doping ( $\mathrm{x}>0$ ), roughly proportional to the number of $\mathrm{Cu}(\mathrm{III})$ sites (Uchida et al., 1991). Spectral density at $\approx 2 \mathrm{eV}$ is transferred down with an isosbestic point at $\approx 1.6 \mathrm{eV}$. The MIR peak is covered by the new absorption with an initial maximum at $0.5 \mathrm{eV}$. At higher doping levels the Drude peak builds up.

The pairs are created in a disproportionation reaction (or Hubbard-U transition (Uchida et al., 1991)) for which the free energy $\Delta \mathrm{G}_{0}$ is the adiabatic Hubbard $\mathrm{U}\left(\mathrm{U}_{\mathrm{ad}}\right)$ :

$$
2 \mathrm{Cu}(\mathrm{II}) \rightarrow \mathrm{Cu}(\mathrm{I})+\mathrm{Cu}(\mathrm{III}) \quad\left(\mathrm{U}_{\mathrm{ad}}=\Delta \mathrm{G}_{0}\right)
$$

Electron pair transfer (EPT) is exchange of the type:

$$
\mathrm{Cu}(\mathrm{III})+\mathrm{Cu}(\mathrm{I}) \rightarrow \mathrm{Cu}(\mathrm{I})+\mathrm{Cu}(\mathrm{III})
$$

with coupled vibrational motion. The obvious condition for SC, except that the atoms of the lattice must be the same, is that the activation energy for the reaction in Equation (3) should tend to zero, which in practical terms means delocalization. Pairing is spontaneous if the free energy $\Delta \mathrm{G}_{0}=\mathrm{U}_{\mathrm{ad}} \leq 0$. If the states which can be reached below a certain temperature correspond to an even number of electrons, the electrons are paired.

\section{Charge Transfer Spectra}

Copper(II) ions with water or oxygen ligands, such as $\mathrm{Cu}\left(\mathrm{H}_{2} \mathrm{O}\right)_{6}{ }^{2+}$, or $\mathrm{Cu}\left(\mathrm{H}_{2} \mathrm{O}\right)_{4}{ }^{2+}$ ions in water solution and the crystal $\mathrm{CuSO}_{4} .5 \mathrm{H}_{2} \mathrm{O}$ are green-blue due to absorption in the red. The highest (singly) occupied orbital has $\mathrm{Cu} 3 \mathrm{~d}\left(\mathrm{x}^{2}-\mathrm{y}^{2}\right)$ symmetry and is strongly mixed with $\mathrm{O} 2 \mathrm{p}$. The blue colour is due to ligand field transitions.

The crystal water may be removed by heating. In $\mathrm{CuSO}_{4}$ the ligand field transitions now appear in the infrared below the visible region, making $\mathrm{CuSO}_{4}$ white. The reason for this may be the strong bonds in the $\mathrm{SO}_{4}{ }^{2-}$ negative ions, making the oxygen bonds to the $\mathrm{Cu}^{2+}$ ion weaker, as compared to the bond between $\mathrm{H}_{2} \mathrm{O}$ and $\mathrm{Cu}^{2+}$.

With crystal water $\mathrm{CuCl}_{2}$ is green, but in pure form $\mathrm{CuCl}_{2}$ and some other salts are brown like the cuprates and $\mathrm{CuO}$, signalling strong absorption in the visible region. We argue here that the latter absorption in the visible region is due to $\mathrm{MM}(\mathrm{CuCu})$ transitions. Typical for $\mathrm{MM}$ charge transfer transitions is that their energy is high if the distance between the metal ions is large. If the distance is as small as about $3 \AA$, the absorption falls in the visible region. This applies to cuprates, $\mathrm{CuO}, \mathrm{CuCl}_{2}$, but not $\mathrm{CuSO}_{4}$. In the latter case the distance between the copper(II) ions is considerably larger than for the first mentioned compounds, making the MM-transitions fall above the visible region.

It may be interesting to compare the Mott oxides $\mathrm{CuO}$ and $\mathrm{NiO}$ (Newman \& Chrenko, 1959). In Ni(II) compounds the MM absorption is at a high energy, which is related to the instability of $\mathrm{Ni}(\mathrm{I})$. However, $\mathrm{Cu}(\mathrm{I})$ is stable due to a filled $3 \mathrm{~d}$-shell and the MM transitions are low.

On the other hand there is no reason to believe that the ligand-metal (LM) charge transfer transitions (O2p $\rightarrow$ $\mathrm{Ni3d}$ ) are lower in $\mathrm{CuO}$ than in $\mathrm{NiO}$. The $\mathrm{LM}$ transitions are above $4 \mathrm{eV}$ in NiO. LM charge transfer energies are

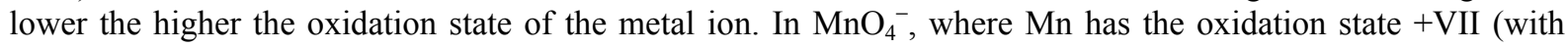
unoccupied d-orbitals), a strong LM-transition appears in the visible part of the spectrum. The same transitions exist in $\mathrm{CrO}_{4}{ }^{2-}$, where $\mathrm{Cr}$ is in the oxidation state $+\mathrm{VI}$, but are higher in energy and red-yellow. $\mathrm{In} \mathrm{TiO}_{2}$, where Ti has oxidation state IV, the LM absorptions appear in the UV region. In cuprates the valence state of copper is only II and the LM charge transfer energy therefore high, probably higher than $4 \mathrm{eV}$.

There is also no reason to believe that metal-ligand (ML) transitions are lower in $\mathrm{Cu}(\mathrm{II})$ than in $\mathrm{Ni}(\mathrm{II})$ compounds. $3 \mathrm{~d} \rightarrow 4 \mathrm{~s}$ transitions are in the ultraviolet $(>3 \mathrm{eV})$ and forbidden, and the allowed $3 \mathrm{~d} \rightarrow 4 \mathrm{p}$ transitions should be another $2 \mathrm{eV}$ higher. In conclusion the LM and ML transitions in cuprates are higher in energy than the ligand field transitions and even the MM transitions. 
Absorption around $2 \mathrm{eV}$ in the cuprates is polarized only in the $\mathrm{CuO}_{2}$ plane (Perkins et al., 1993; Falck, Levy, Kastner, \& Birgeneau, 1992) as expected from MM transitions in the $\mathrm{CuO}_{2}$ plane. $\mathrm{LM}$ and ML transitions are polarized in all directions. Hence the gap at $1.7-2.5 \mathrm{eV}$ is due to $\mathrm{MM}$ transitions (Hubbard- $\mathrm{U}_{\text {vert }}$ ).

Part of the misunderstanding regarding $\mathrm{LM}$ charge transfer spectra arises from a paper which shows the same satellite structure of $\mathrm{La}_{2-\mathrm{x}} \mathrm{Sr}_{\mathrm{x}} \mathrm{CuO}_{4}$ and $\mathrm{YBa}_{2} \mathrm{Cu}_{3} \mathrm{O}_{6.7}$ as for other $\mathrm{Cu}^{2+}$ systems (Fujimori, Takayama-Muromachi, Uchida, \& Okai, 1987). The result is correctly interpreted as due to a more attractive $\mathrm{Cu}$ site after $2 \mathrm{p}$-ionization, according to the Sawatzky-Larsson model (Larsson, 1976). The Cu2p-hole is mainly screened by increased $3 \mathrm{~d}$-character in the occupied Cu3d-O2p molecular orbitals. The satellite/main line energy gap is equal to the LM energy gap, and it remains at about $4 \mathrm{eV}$ after $\mathrm{Cu} 2 \mathrm{p}$ ionization (Fujimori, Takayama-Muromachi, Uchida, \& Okai, 1987). In any case Cu2p ionization is not involved in SC.

\section{The Marcus Model for Cuprates}

The bondlengths between the atoms at a site depends on the number of electrons present. To every local state belongs a set of bondlengths and bondangles. A $\mathrm{Cu}(\mathrm{III})$ site with empty $\mathrm{Cu}-3 \mathrm{~d}\left(\mathrm{x}^{2}-\mathrm{y}^{2}\right)-\mathrm{O} 2 \mathrm{p}$ antibonding molecular orbital has a shorter (by about $0.1 \AA$ ) $\mathrm{CuO}$ bond than a $\mathrm{Cu}(\mathrm{II})$ site, which contains one electron in this orbital. A typical case is single electron exchange [Figure 1(a)]. The exchange rate varies depending on activation barrier. Activation barriers are large or small depending on bondlength differences. Vibrations, bondlengths and activation barriers are coupled.

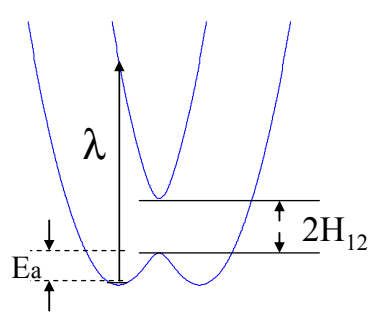

(a)

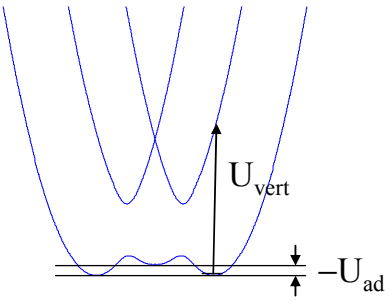

(b)

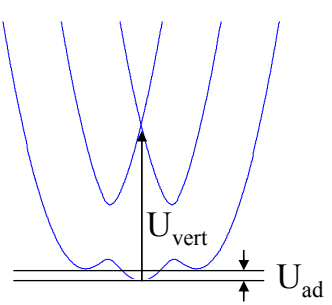

(c)

Figure 1. Potential energy surfaces in the Marcus model. (a) one-electron transfer; reorganization energy $(\lambda)$, coupling $\left(2 \mathrm{H}_{12}\right)$ and activation energy $\left(\mathrm{E}_{\mathrm{a}}\right)$ are shown; (b,c) electron pair transfer. $\mathrm{U}_{\text {vert }}$ and $\mathrm{U}_{\mathrm{ad}}$ are shown. (b) negative $U$ case $\left(\mathrm{U}_{\mathrm{ad}}<0\right)$ and (c) positive $\mathrm{U}$ case $\left(\mathrm{U}_{\mathrm{ad}}>0\right)$

Disproportionation energy is equivalent to adiabatic Hubbard U:

$$
2 \mathrm{Cu}(\mathrm{II}) \rightarrow \mathrm{Cu}(\mathrm{I})+\mathrm{Cu}(\mathrm{III}) \quad\left(\Delta \mathrm{G}_{0}=\mathrm{U}_{\mathrm{ad}}\right)
$$

The vibrational breathing or half breathing mode is coupled to $\mathrm{CuO}$ bondlength, in its turn coupled to disproportionation, as indicated in Figure 1. Figure 1(b) is the spontaneous reaction with $U_{a d}<0$, while Figure 1(c) shows the case typical for cuprates with $U_{a d}>0$.

Heikes and Johnston were the first to make a systematic study (Heikes \& Johnston, 1957) of the Mott insulators $\mathrm{MnO}, \mathrm{CoO}, \mathrm{NiO}$, and $\mathrm{CuO}$. $\mathrm{Cu}(\mathrm{III})$ sites were created by doping with $\mathrm{Li}_{2} \mathrm{O}$. The cuprates have a similar activated conductivity if slightly doped. Apparently localization occurs in cuprates as well as in $\mathrm{CuO}$, and this is consistent with the existence of stripes (Tranquada, Sternlieb, Axe, Nakamura, \& Uchida,1995; Tranquada et al., 2004). Cuprates are polarons rather than semiconductors and have rich absorption spectrum in the "bandgap" (Perkins et al., 1993; Perkins, Birgeneau, Graybeal, Kastner, \& Kleinberg, 1998). Oxidative doping of cuprates changes some $\mathrm{Cu}(\mathrm{II})$ to $\mathrm{Cu}(\mathrm{III})$ sites in the $\mathrm{CuO}_{2}$ plane. Conductivity with the low activation energy of about $0.035 \mathrm{eV}$ appears (Preyer et al., 1989), due to single electron exchange. The conductivity is

$$
\mathrm{I} \sim \mathrm{v}_{\mathrm{n}} \cdot \kappa \cdot \exp \left(-\frac{\mathrm{E}_{\mathrm{a}}}{\mathrm{k}_{\mathrm{B}} \mathrm{T}}\right) \quad \text { where } \quad \kappa=\frac{\Delta^{2}}{4 \pi \hbar}\left(\frac{\pi^{3}}{\lambda \mathrm{k}_{\mathrm{B}} \mathrm{T}}\right)^{1 / 2}
$$

$E_{a}$ is the activation energy, $\kappa$ the electronic transmission factor, and $v_{n}$ the vibration frequency of the activating motion, $\lambda$ is reorganization energy and $\Delta$ gap energy (Brunschwig, Logan, Newton, \& Sutin, 1980). Equation (5) 
has a peak in the conductivity, typical for hopping. The T-dependence of $\kappa$ originates from the Landau-Zener avoided crossing problem (Landau, 1932).

The reorganization energy $(\lambda)$ is also the vertical excitation energy from the $\mathrm{Cu}^{2+} / \mathrm{Cu}^{3+}$ to the $\mathrm{Cu}^{3+} / \mathrm{Cu}^{2+}$ energy surfaces (Figure 1a). This is very likely the origin of the $0.13 \mathrm{eV}$ excitation measured by Falck, Levy, Kastner, and Birgeneau (1993) for $\mathrm{La}_{2} \mathrm{CuO}_{4+\mathrm{y}}$. The thermal activation energy is only $1 / 4$ of the excitation energy in the Marcus model, or $1 / 4 \cdot 0.13 \approx 0.033$ (Figure 1a).

Ono et al. have made a fitting to the Hall coefficient $\mathrm{R}_{\mathrm{H}}$ (Ono, Komiya, \& Ando, 2007; Timusk \& Statt, 1999; Dzhumanov, Ganiev, \& Djumanov, 2014; Hazra, Mandal, \& Ghosh, 1996) of a similar type as in Equation (5), but with two activation energies: $\mathrm{E}_{\mathrm{a}}^{(1)}=0.0435 \mathrm{eV}$ and $\mathrm{E}_{\mathrm{a}}^{(2)}=0.445 \mathrm{eV}$. According to the Marcus model the optical excitation energy is four times as much, i.e. $U_{\text {vert }}^{(1)}=0.174 \mathrm{eV}$ and $\mathrm{U}_{\text {vert }}^{(2)}=1.78 \mathrm{eV}$. We may interpret this as follows: The lower activation energy is due to $\mathrm{Cu}(\mathrm{II}) / \mathrm{Cu}(\mathrm{III})$ exchange and the higher to charge transfer of disproportionation type (Hubbard $\mathrm{U}_{\text {vert }}$ ), followed by electron pair transfer

As a function of temperature (T) resistivity is infinite for $T=0$, goes through a minimum $\left(T^{*}\right)$ and then increases as for a metal, for increasing T (Takagi et al., 1992; Hwang et al., 1994). If the system is superconducting for $\mathrm{T}<\mathrm{T}_{\mathrm{C}}$, the resistivity in the $\mathrm{CuO}_{2}$ plane quickly rises for $\mathrm{T}>\mathrm{T}_{\mathrm{C}}$, goes through a maximum, and then drops to a minimum before it rises in the same way as for a metal. The resistivity $\rho_{a b}$ thus shows a maximum between $T_{C}$ and $\mathrm{T}^{*}$.

A very small increase of doping level may decrease resistivity from infinity to zero, as $T \rightarrow 0$ (Takagi et al., 1992; Hwang et al., 1994). Above and below the critical level, the resistivity curves run parallel from large $\mathrm{T}$ down to $\mathrm{T}^{*}$. Already at $\mathrm{T}^{*}$ the resistivity of the superconductor starts to deviate but is still close to the underdoped curve until a maximum. The minimum at $\mathrm{T}^{*}$ is considered to be due to opening of the pseudogap. Apparently there are two different mechanisms for conductivity. One has to do with activation of single electron hopping conductivity, tending to metallic above $\mathrm{T}^{*}$. The other leads to a superconducting gap as $\mathrm{T} \rightarrow 0$. We may conclude that the mechanism for SC is very different from the mechanism for metallic conductivity or electron hopping. The former is due to electron pair transfer (bosonic behaviour) and the latter to electron transfer $[\mathrm{Cu}(\mathrm{II}) / \mathrm{Cu}(\mathrm{III})]$ exchange (fermionic behaviour). Small energy differences determine the statistics.

In the Marcus model for electron pairs there are three energy surfaces of near parabolic shape. The ground state for cuprates is known to be a spin coupled, antiferromagnetic phase in the case of cuprates of type $\mathrm{Cu}(\mathrm{II}) / \mathrm{Cu}(\mathrm{II})$. Two higher states are charged states of the type $\mathrm{Cu}(\mathrm{I}) / \mathrm{Cu}(\mathrm{III})$ and $\mathrm{Cu}(\mathrm{III}) / \mathrm{Cu}(\mathrm{I})$. A major difference to the traditional Marcus case is that we need the Hubbard-U parameter for the difference in total free energy between charged state and the spin-coupled state.

The large width of the MM state follows from the fact that the final state is the strongly sloping site of the charged state parabola (Figure 1c). Electrochemical potentials for disproportionation reactions have been published for a number of systems as "Frost diagrams". More information and references can be found on Internet. Unfortunately $\mathrm{Cu}(\mathrm{III})$ is unstable in water solvent, and hence there may be scarce experimental data regarding the redox potential for the case of disproportionation of $\mathrm{Cu}(\mathrm{II})$.

We conclude from Figs. $1 \mathrm{~b}$ and $1 \mathrm{c}$ that there are more than one way to define Hubbard-U: The vertical excitation energy, $U_{\text {vert }}$, and the adiabatic energy difference in the equilibrium points of the total energy parabolas, $U_{a d}$ are different by nearly $2 \mathrm{eV}$.

In summary Marcus type models, particularly the Jortner version (Kestner, Logan, \& Jortner, 1974; Jortner, 1976; Jortner \& Bixon, 1969; Brunschwig, Logan, Newton, \& Sutin, 1980), tells us which vibrations or phonons are coupled to electron pair transfer ("the electron-lattice polarization effect") (Takagi et al., 1992; Hwang et al., 1994; Moskvin, 2011). Since the Marcus parabolas are total energies, the change of curvature at curve crossings reveals itself in vibration spectra ("dynamic charge inhomogeneity") ((Takagi et al., 1992; Hwang et al., 1994)), and in time resolved spectra (non-linear lattice dynamics) (Moskvin, 2011). Since the weak dependence of $T$ in $\kappa$ can be ignored, Equation (5) shows that the dependence of $\mathrm{T}$ is essentially exponential. The Jortner model, on the other hand, uses vibronic energy levels for tunnelling of the nuclei through the activation barrier. For very low T there remains one level for tunnelling (Kestner, Logan, \& Jortner, 1974; Jortner, 1976; Jortner \& Bixon, 1969; Brunschwig, Logan, Newton, \& Sutin, 1980), leading to slower decrease of rate.

\section{Hubbard-U}

In Mott's interpretation Hubbard $U$ is excitation energy over a charge transfer transition from metal to the adjacent metal atom ((Kestner, Logan, \& Jortner, 1974; Jortner, 1976; Jortner \& Bixon, 1969; Brunschwig, Logan, Newton, \& Sutin, 1980). However, the atomic integral does not approximate the charge transfer energy. 
In semi-empirical quantum calculations, where $\phi$ is an approximation to an atomic orbital, the value of the integral in Equation (6) is $15-20 \mathrm{eV}$ for transition metal ions or carbon atoms. Values in this range using atomic ab initio methods have been published for cuprates, but they are certainly meaningless. The energy of charge-transfer excitations have a number of negative contributions (Larsson, 2013).

$$
\mathrm{U}=\int \phi^{*}(1) \phi^{*}(1) \frac{1}{\mathrm{r}_{12}} \phi(2) \phi(2) \mathrm{dV}
$$

If Equation (6) is adopted, obviously $U>0$. Mott improved Hubbard-U by defining $U$ as the difference between ionization energy I and electron affinity (Mott, 1968), not included in Equation (6):

$$
\mathrm{U}=\mathrm{I}-\mathrm{A}
$$

Even with this definition $U>0$ in most cases. By Equation (7) $U$ is the energy for removing an electron from one site and putting it on another site, so far away that the attractive energy between the positive hole and negative electron can be neglected (Mott, 1968). The intention of Mott was obviously to obtain a simple measure of the charge transfer energy in an oxide or salt. It is unclear why he did not want to include the electron-hole attraction, which is simply $1 / \mathrm{R}$ Hartree (atomic units), if the distance between hole and electron after charge transfer is $\mathrm{R}$ Bohr $=\mathrm{R} \cdot 1.89 \AA$. Since the electron-hole interaction is in fact large, the electron avoids transferring a long distance in an actual experiment (for example in a solid state charge transfer transition, in a photosynthetic charge separation or in the creation of static electricity). If the distance between the hole and the electron is R, one at least has to improve Equation (7) to the acceptable definition:

$$
\mathrm{U}_{\mathrm{ad}}=\mathrm{I}-\mathrm{A}-1 / \mathrm{R} \quad \text { (atomic units) }
$$

$\mathrm{R} \rightarrow \infty$ is unrealistic. There are additional corrections to Equation (8), however. In fact $\mathrm{U}$ has to be understood as a free energy, and then the only possible equation is

$$
\mathrm{U}_{\mathrm{ad}}=\text { DPE (disproportionation energy) }
$$

DPE can be obtained from electrochemical data in fortunate cases. Seldom $U_{a d}$ exceeds a fraction of an eV, and is frequently negative. Obviously $\mathrm{U}_{\mathrm{ad}}<0$ for a "missing" valence state such as $\mathrm{Tl}^{2+}$, which disproportionates into $\mathrm{Tl}^{+}$and $\mathrm{Tl}^{3+}$, contradicting eqs.(6,7). Generally the negative "lattice enthalpy" has to be added (Larsson, 2013), since there is always attraction between the positive hole and the negative electron (Born, 1919; Haber, 1919).

Doping introduces attractive $\mathrm{Cu}(\mathrm{III})$ sites, making $\mathrm{U}_{\mathrm{ad}}$ negative and the charged phase eventually stable (Larsson, 2013). SC occurs in the interphase (Larsson, 2012, 2013) between charged and spin coupled phase.

\section{Assignments in Visible and IR Spectra}

The large gap below $1.7 \mathrm{eV}$ in pure cuprates (Larsson, 2013) is easy to explain in a local picture, since the only possibility for electronic states are ligand field states and $\mathrm{MM}$ charge transfer states. Both start above $1.7 \mathrm{eV}$, but only the latter are allowed. For historical reasons the MM-transitions are called Mott-Hubbard states (Mott, 1968). The strong absorption is thus due to electron transfer $a \rightarrow b$ between adjacent sites A and B with equal valence:

$$
\mathrm{h} v+2 \mathrm{Cu}(\mathrm{II}) \rightarrow \mathrm{Cu}(\mathrm{I})+\mathrm{Cu}(\mathrm{III}) \quad\left(\mathrm{h} v=\mathrm{U}_{\text {vert }}\right)
$$

The energy is increasing with transfer distance, but the transition moment is decreasing. $a$ and $b$ are $\mathrm{Cu} 3 \mathrm{~d}\left(\mathrm{x}^{2}-\mathrm{y}^{2}\right)$ orbitals (same spin and strong ligand admixtures) on adjacent sites A and B, respectively. Tokura et al. compared optical conductivity in a number of cuprates (Tokura et al., 1990), and tried to correlate peak maximum at 1.5 - 2 $\mathrm{eV}$ with structure. The reason for the quite large width is obvious from the Marcus model. The energy of the charge transfer state is parabolic, but with equilibrium distance different from the one of the ground state. It follows that the energy of the final state is strongly sloping (Figures 1,2). The large width is thus due to the (half)-breathing vibrations of the Marcus model.

There are two nearly parabolic energy surfaces with different equilibrium points, corresponding to spin-coupled $(a b+b a)$ and charged $a a, b b$, forming a spin-coupled ground state and excited charged states, respectively. The created $\mathrm{Cu}(\mathrm{I}) / \mathrm{Cu}(\mathrm{III})$ pair relaxes its geometry along the left parabola of Figure 2 . After doping there are $\mathrm{Cu}(\mathrm{III})$ sites which may be regarded as deeper holes for the electrons.

Perkins et al. studied optical absorption in several undoped cuprates $(\mathrm{x}=0)$ with all copper atoms in the valence state II, and found weak absorption in the MIR region with polarization in the $\mathrm{CuO}_{2}$ plane (Perkins et al., 1993). The sidebands have been interpreted in different ways. Here a different interpretation is suggested, where the weak absorption is due to vibrational excitation that obtains intensity due to the influence of the charge transfer 
state on the ground state energy surface (Figure 2). Below the avoided crossing the vibrational levels of the charged state perturb slightly the vibrational levels of the ground state, making the transition slightly allowed.

The transition momentet for $\mathrm{x}$-polarized light is:

$$
\mu_{\mathrm{x}}(\mathrm{Q})=\left\langle\phi_{\mathrm{i}}|\mathrm{x}| \phi_{\mathrm{a}}\right\rangle \cdot\left\langle\Xi_{\mathrm{iv}} \mid \Xi_{\mathrm{au}}\right\rangle
$$

In the present case the orbitals $\phi_{i}$ and $\phi_{a}$ are $3 \mathrm{~d}\left(\mathrm{x}^{2}-\mathrm{y}^{2}\right)$ molecular orbitals at adjacent sites. They overlap through the oxygen $2 p_{\mathrm{x}}$ or $2 \mathrm{p}_{\mathrm{y}}$ orbitals. $\mu_{\mathrm{x}}$ and $\mu_{\mathrm{y}}$, but not $\mu_{\mathrm{z}}$, are different from zero for orbitals in the $\mathrm{CuO}_{2}$ plane. The vibrational levels and the electronic levels together forming vibronic levels, and borrowing intensity from the other, higher parabola, thereby making some higher vibrational levels weakly allowed.

In Equation (11) $\left\langle\Xi_{\mathrm{iv}} \mid \Xi_{\mathrm{au}}\right\rangle$ is the vibrational overlap between vibrational states $\mathrm{v}$ and $\mathrm{u}$ belonging to the $\mathrm{i}$ and a electronic states, respectively. Since these states belong to different electronic states the overlaps occur particularly in the region where the two parabolas in Figure 2 are intersecting. Very likely this is the reason for the non-zero intensity for the MIR transitions, and explains the correlation between the MIR absorptions at 0.4 and the absorption at $1.7 \mathrm{eV}$. The MIR absorption is of MM-type and the electronic interaction between the energy curves is due to electronic overlap via oxygen $\mathrm{O} 2 \mathrm{p}$.

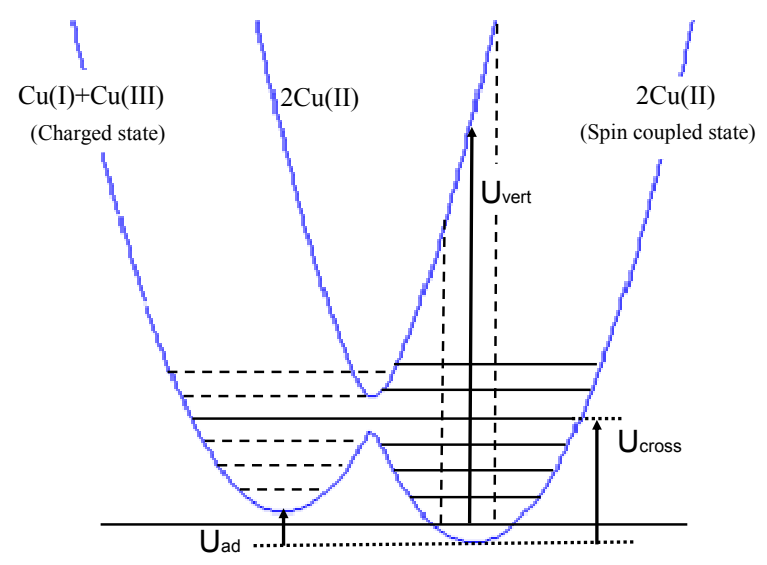

Figure 2. Marcus model for electron pairs. Dashed vertical lines demonstrate the width of the absorption at $\approx 2$ eV. Full lines: Vibronic levels. Perturbing vibrational levels of the charged state are dashed

Uchida et al. (1991) demonstrated transfer of spectral density to lower energies after doping, as already mentioned. Absorption increases fast with doping in the whole region below $1 \mathrm{eV}$. For $6 \%$, as compared to $2 \%$ doping, Uchida et al. (1991) find a further move of the spectral density to even lower energies, ending up in the Drude peak.

How are we going to explain that spectral density, polarized in the $\mathrm{CuO}_{2}$ plane, upon doping is moved from the large absorption peak at $\approx 1.7 \mathrm{eV}$ to the region below $1.5 \mathrm{eV}$, and most of it below $1 \mathrm{eV}$ (Uchida et al., 1991)? Both the strong absorption around $2 \mathrm{eV}$ and the weaker one below $1.5 \mathrm{eV}$ are polarized in the $\mathrm{CuO}_{2}$ plane, and this together with high intensity shows that we are dealing with MM charge transfer transitions. The ligand field transitions whose intensity can be neglected compared to the MM transitions, may be one reason for the less than perfect isosbestic point.

The transfer of spectral density is not restricted to cuprates. Katsufuji, Okimoto, and Tokura (1995) show that varying $\mathrm{x}$ from 0 to 1 in $\mathrm{R}_{1-\mathrm{x}} \mathrm{Ca}_{\mathrm{x}} \mathrm{TiO}_{3}$, where $\mathrm{R}=\mathrm{La}, \mathrm{Nd}$, Sm, or $\mathrm{Y}$, moves spectral density from the region above $1 \mathrm{eV}$ to the region below $1 \mathrm{eV}$. For $\mathrm{x}=0$ there are only $\mathrm{Ti}(\mathrm{III})$ sites (one $3 \mathrm{~d}$ electron per Ti); for $\mathrm{x}=1\left(\mathrm{CaTiO}_{3}\right)$, only $\mathrm{Ti}(\mathrm{IV})$ sites with no Ti3d electrons. If there are only $\mathrm{Ti}(\mathrm{III})$ sites, disproportionation to $\mathrm{Ti}(\mathrm{II}) / \mathrm{Ti}(\mathrm{IV})$ is possible at excitation, with Hubbard $\mathrm{U}_{\text {vert }} \approx 2 \mathrm{eV}$. After Ti(IV) sites have appeared for $\mathrm{x}>0$, vertical electron exchange reactions at a low energy become possible (Figure 1a), but Ti(II) sites start to disappear. Weak absorptions below the $\mathrm{MM}$ transition energy $\left(\mathrm{U}_{\text {vert }}\right)$ are quite common in transition metal oxides after doping, and indicate a possibility for SC. Whether or not SC appears is controlled by Hubbard- $\mathrm{U}_{\mathrm{ad}}$. 
Thus when $\mathrm{Cu}(\mathrm{III})$ sites become available in $\mathrm{La}_{2-\mathrm{x}} \mathrm{Sr}_{\mathrm{x}} \mathrm{CuO}_{4}(\mathrm{x}>0)$ there are many new possibilities for photo-induced charge transfer, for example of the type:

$$
\mathrm{h} v+\mathrm{Cu}(\mathrm{II})+\mathrm{Cu}(\mathrm{III}) \leftrightarrow \mathrm{Cu}(\mathrm{III})+\mathrm{Cu}(\mathrm{II})
$$

This is one reason for transfer of spectral density to the lower energy range.

Also transitions of the form seen in Equation (10) are possible at a lower energy, and these are connected to the pseudogap. The pseudogap has been discussed in earlier publications (Larsson, 2010, 2012). The active orbitals are $\mathrm{Cu} 3 \mathrm{~d}\left(\mathrm{x}^{2}-\mathrm{y}^{2}\right)$ mixed with $\mathrm{O} 2 \mathrm{p}(\mathrm{x})$ and $\mathrm{O} 2 \mathrm{p}(\mathrm{y})$. If this orbital is called $a$ on site $\mathrm{A}$ and $b$ on site $\mathrm{B}$, the hole state is $a b+b a$ and forms the spin-coupled ground state of undoped cuprates. The hole states $a a$ and $b b$ are the charged states. The ground state is a superposition of $a b+b a$ and $a a+b b$. Without doping the $a a+b b$ state is $1.7-2.5 \mathrm{eV}$ vertically above the ground state $a b+b a$, but only $\approx 0.2 \mathrm{eV}$ in an adiabatic process. The activation energy for creating $a a+b b$ and $a a-b b$ in a thermal process should be $\approx 1 / 4 \cdot 2 \mathrm{eV}$ in the Marcus model, if $\mathrm{U}_{\mathrm{ad}}$ is neglected.

The pseudogap is due to the transition $a b+b a \rightarrow a a-b b$ (Larsson, 2010). In the superconducting region, the mixing of $a b+b a$ and $a a+b b$ forms the ground state and the superconducting gap to $a a-b b$. With less doping this gap becomes the pseudogap and can no support SC because of the term $a b+b a$ (Larsson, 2010, 2012). The pseudogap is lower in energy but becomes increasingly visible as the number of charges in the $\mathrm{CuO}_{2}$ plane is increased. The charged energy curve in the Marcus model, Figs. 1b,c and Figure 2, lower its energy compared to the spin-coupled state (Larsson, 2012), due to the increased number of $\mathrm{Cu}$ (III) sites.

\section{Discussion}

Cuprates are different from other copper compounds by the existence of square planar $\mathrm{CuO}_{2}$ planes where the number of electrons per site can be changed by doping. This makes three phases possible, a charged phase, an antiferromagnetic phase and a superconducting phase (Larsson, 2012). The former two are insulating, but may interact and form a superconducting phase (Larsson, 1986; Basov, \& Timusk, 2005). The ground state without doping is the anti-ferromagnetically ordered phase. With slight oxidative doping $\mathrm{Cu}$ (III) sites appear among the $\mathrm{Cu}$ (II) sites. New quantum mechanical states appear, of the following type:

1). The local $\mathrm{Cu}$ (III) site can interact with $\mathrm{Cu}(\mathrm{II})$ sites by electron exchange at the same or slightly different energy. For each pair of $\mathrm{Cu}(\mathrm{III}) / \mathrm{Cu}$ (II) sites there is now another electronic state of the type $\mathrm{Cu}(\mathrm{II}) / \mathrm{Cu}(\mathrm{III})$. To this new situation the Marcus model is a very useful model, which is complementary to ligand field theory for single sites.

In a localized mixed-valence system it is legitimate to use a localized theory and use concepts such as charge transfer states and charge transfer gap. The $\mathrm{Cu}(\mathrm{II}) / \mathrm{Cu}(\mathrm{III})$ interaction puts its marks on the absorption and photoconductivity spectra, and is very likely responsible for the absorption at $0.13 \mathrm{eV}$.

2). The pseudogap is the gap formed between an inter-site two-electron state due to interaction between the hole states $a b+b a$ and $a a+b b$ and the $a a-b b$ excited state. For decreasing Hubbard U, the $a a+b b$ component in the ground state is increased compared to the $a b+b a$ component. The ground state tends to $a a+b b$ when $\mathrm{U}_{\mathrm{ad}}$ turns negative and the gap is then to $a a-b b$. Only paired states are now present at low energy and the system may become superconducting and follow Bose-Einstein statistics.

3). Vibrational levels which borrow intensity from charged states. In the "final" interactions a superconducting phase with a vibronic ground state is formed with concerted motion of electrons and nuclei, in a similar way as in the Cooper model (Cooper, 1956).

The vertical gap, $\mathrm{U}_{\text {vert }}$, is $\approx 2 \mathrm{eV}$ and the adiabatic gap, $\mathrm{U}_{\mathrm{ad}}$, only $\approx 0.2 \mathrm{eV}$. This may be considered anomalous compared to the corresponding values for $\mathrm{NiO}$, and depends on the fact that copper has three stable oxidation states $\mathrm{Cu}(\mathrm{I}), \mathrm{Cu}(\mathrm{II})$, and $\mathrm{Cu}(\mathrm{III})$.

At oxidative doping of the pure cuprate the absorption at $2 \mathrm{eV}$ is decreased. The reason is simply that the average energy needed to move the electron to the other site is decreased with an increased number of $\mathrm{Cu}(\mathrm{III})$ sites. The maximum value is $\approx 2 \mathrm{eV}$ without doping, since all other sites are $\mathrm{Cu}(\mathrm{II})$ sites. When holes are created, so that the $\mathrm{Cu}$ (III) sites are formed, the energy spectrum is completely changed. It is possible to excite into the hole or to sites adjacent to the holes.

Hubbard- $U$ is a free energy and has to be calculated as a free energy. The only existing, accurate calculation (as far as I know) has lead to a value of $U_{\text {ad }}$ close to zero (Klimkans, 2001). The most common shortcoming is that the cluster has been too small (sometimes just the copper ions), that correlation effects have not been included, or that Coulomb fields have not been included properly. 
The assignment of spectra given here shows the importance of structural differences between different oxidation states. This structural dependence is also of decisive importance in the nuclear dynamics of excited states and electron transfer (Reznik et al., 2006; Mankowsky et al., 2014). These problems will be discussed in forthcoming publications.

\section{Conclusion}

SC depends on electron pairing on $\mathrm{Cu}$ sites and successive, activationless electron pair transfer between the sites. It develops in a localized system under the conditions that make $\mathrm{U} \rightarrow 0$. Hole-doped cuprates are good examples that doping tends to make the $\mathrm{Cu}^{+} / \mathrm{Cu}^{3+}$ charged phase more stable and hence make $\mathrm{U} \rightarrow 0$ (Larsson, 2013). Configuration interaction between the insulating charged and spin-coupled configurations leads to a superconducting state.

\section{References}

Basov, D. N., \& Timusk, T. (2005). Electrodynamics of high-T c superconductors. Reviews of modern physics, 77(2), 721. http://dx.doi.org/10.1103/RevModPhys.77.721

Bednorz, J. G., \& Müller, K. A. (1986). Possible highT c superconductivity in the $\mathrm{Ba}-\mathrm{La}-\mathrm{Cu}-\mathrm{O}$ system. Zeitschrift für Physik B Condensed Matter, 64(2), 189-193. http://dx.doi.org/10.1007/BF01303701

Born, M. (1919). Verhandlungen der Deutschen physikalischen Gesellschaft, 21, 13.

Brunschwig, B. S., Logan, J., Newton, M. D., \& Sutin, N. (1980). A semiclassical treatment of electron-exchange reactions. Application to the hexaaquoiron (II)-hexaaquoiron (III) system. Journal of the American Chemical Society, 102(18), 5798-5809. http://dx.doi.org/10.1021/ja00538a017

Cooper, L. N. (1956). Bound electron pairs in a degenerate Fermi gas. Physical Review, 104(4), 1189-1190. http://dx.doi.org/10.1103/PhysRev.104.1189

de Graaf, C., \& Broer, R. (2000). Midinfrared spectrum of undoped cuprates: dd transitions studied by ab initio methods. Physical Review B, 62(1), 702-709. http://dx.doi.org/10.1103/PhysRevB.62.702

Dzhumanov, S., Ganiev, O. K., \& Djumanov, S. S. (2014). Normal-state conductivity of underdoped to overdoped cuprate superconductors: Pseudogap effects on the in-plane and c-axis charge transports. Physica B: Condensed Matter, 440, 17-32. http://dx.doi.org/10.1016/j.physb.2014.01.017

Falck, J. P., Levy, A., Kastner, M. A., \& Birgeneau, R. J. (1992). Charge-transfer spectrum and its temperature dependence in $\mathrm{La} 2 \mathrm{CuO}$ 4. Physical review letters, 69(7), 1109. http://dx.doi.org/10.1103/PhysRevLett.6 9.1109

Falck, J. P., Levy, A., Kastner, M. A., \& Birgeneau, R. J. (1993). Optical excitation of polaronic impurities in La $2 \mathrm{CuO} 4+$ y. Physical Review B, 48(6), 4043-4046. http://dx.doi.org/10.1103/PhysRevB.48.4043

Fujimori, A., Takayama-Muromachi, E., Uchida, Y., \& Okai, B. (1987). Spectroscopic evidence for strongly correlated electronic states in $\mathrm{La}-\mathrm{Sr}-\mathrm{Cu}$ and $\mathrm{Y}-\mathrm{Ba}-\mathrm{Cu}$ oxides. Physical Review B, 35(16), 8814. http://dx.doi.org/10.1103/PhysRevB.35.8814

Haber, F. (1919). Verhandlungen der Deutschen physikalischen Gesellschaft, 21.,750.

Hazra, S., Mandal, S., \& Ghosh, A. (1996). Transport mechanism in nonconventional bismuth cuprate glass. The Journal of chemical physics, 104(24), 10041-10045. http://dx.doi.org/10.1063/1.471748

Heikes, R. R., \& Johnston, W. D. (1957). Mechanism of Conduction in Li - Substituted Transition Metal Oxides. The Journal of Chemical Physics, 26(3), 582-587. http://dx.doi.org/10.1063/1.1743350

Herr, S. L., Kamarás, K., Porter, C. D., Doss, M. G., Tanner, D. B., Bonn, D. A., ... \& Timusk, T. (1987). Optical properties of $\mathrm{La} 1.85 \mathrm{Sr} 0.15 \mathrm{CuO} 4$ : Evidence for strong electron-phonon and electron-electron interactions. Physical Review B, 36(1), 733-735. http://dx.doi.org/10.1103/PhysRevB.36.733

Hush, N. S. (1967). Intervalence-transfer absorption. Part 2. Theoretical considerations and spectroscopic data. Prog. Inorg. Chem, 8(391), 12. http://dx.doi.org/10.1002/9780470166093.ch7

Hwang, H. Y., Batlogg, B., Takagi, H., Kao, H. L., Kwo, J., Cava, R. J., ... \& Peck Jr, W. F. (1994). Scaling of the temperature dependent Hall effect in La 2- x Sr x CuO 4. Physical review letters, 72(16), 2636-2639. http://dx.doi.org/10.1103/PhysRevLett.72.2636

Jortner, J. (1976). Temperature dependent activation energy for electron transfer between biological molecules. The Journal of Chemical Physics, 64(12), 4860-4867. http://dx.doi.org/10.1063/1.432142 
Jortner, J., \& Bixon, M. (1969). Radiationless transitions in polyatomic molecules. Israel Journal of Chemistry, 7(2), 189-220. http://dx.doi.org/10.1002/ijch.196900032

Kastner, M. A., Birgeneau, R. J., Shirane, G., \& Endoh, Y. (1998). Magnetic, transport, and optical properties of monolayer copper oxides. Reviews of Modern Physics, 70(3), 897-928. http://dx.doi.org/10.1103/RevMod Phys. 70.897

Katsufuji, T., Okimoto, Y., \& Tokura, Y. (1995). Spectral weight transfer of the optical conductivity in doped Mott insulators. Physical review letters, 75(19), 3497-3500. http://dx.doi.org/10.1103/PhysRevLett.75.3497

Kestner, N. R., Logan, J., \& Jortner, J. (1974). Thermal electron transfer reactions in polar solvents. The Journal of Physical Chemistry, 78(21), 2148-2166. http://dx.doi.org/10.1021/j100614a017

Klimkans, A. (2001). Quantum Mechanical Studies of Electron Transport Processes Including Superconductivity. Chalmers University of Technology.

Klimkāns, A., \& Larsson, S. (2000). Vibrational model for electron transfer in the limit of small activation barriers. International Journal of Quantum Chemistry, 77(1), 211-220. http://dx.doi.org/10.1002/(SICI) 1097-461X(2000)77:1<211::AID-QUA20>3.0.CO;2-C

Klimkans, A., \& Larsson, S. (2001). Electronic structure of a copper (III) compound. Journal of Chemical Physics, 115(1), 466-471. http://dx.doi.org/10.1063/1.1377878

Landau, L. D. (1932). On the theory of transfer of energy at collisions II. Phys. Z. Sowjetunion, 2(46), 7.

Larsson, S. (1976). Shake-up and multiplet structure of ESCA satellites of $\mathrm{Cu}$ compounds. Chemical Physics Letters, 40(3), 362-366. http://dx.doi.org/10.1016/0009-2614(76)85097-X

Larsson, S. (1981). Electron transfer in chemical and biological systems. Orbital rules for nonadiabatic transfer. Journal of the American Chemical Society, 103(14), 4034-4040. http://dx.doi.org/10.1021/ja00404a010

Larsson, S. (1986). Electron transport in condensed matter. A novel approach using quantum chemical methods. International journal of quantum chemistry, 30(1), 31-49. http://dx.doi.org/10.1002/qua.560300106

Larsson, S. (1989). Localization condition for metallic conductivity and superconductivity. Chemical Physics Letters, 157(5), 403-408. http://dx.doi.org/10.1016/0009-2614(89)87271-9

Larsson, S. (2002). Chemical model for superconductivity. International journal of quantum chemistry, 90(4 - 5), 1457-1469. http://dx.doi.org/10.1002/qua.10423

Larsson, S. (2010). Charge transfer transitions in cuprates. Chemical Physics Letters, 491(1), 49-53. http://dx.doi.org/10.1016/j.cplett.2010.03.076

Larsson, S. (2012). Microscopic theory for insulator to metal transition in cuprates. International Journal of Quantum Chemistry, 112(7), 1829-1837. http://dx.doi.org/10.1002/qua.23207

Larsson, S. (2013). Lattice Enthalpy Drives Hubbard U to Zero. Journal of Modern Physics, 4(6A), 29-32 http://dx.doi.org/10.4236/jmp.2013.46A007

Mankowsky, R., Subedi, A., Först, M., Mariager, S. O., Chollet, M., Lemke, H. T., ... \& Cavalleri, A. (2014). Nonlinear lattice dynamics as a basis for enhanced superconductivity in $\mathrm{YBa} 2 \mathrm{Cu} 3 \mathrm{O} 6$. 5. Nature, 516(7529), 71-73. http://dx.doi.org/10.1038/nature13875

Marcus, R. A. (1964). Chemical and electrochemical electron-transfer theory. Annual Review of Physical Chemistry, 15(1), 155-196. http://dx.doi.org/10.1146/annurev.pc.15.100164.001103

Moskvin, A. S. (2011). What is the true charge transfer gap in parent insulating cuprates? arXiv:1105.3159v1.

Mott, N. F. (1968). Metal-insulator transition. Reviews of Modern Physics, 40(4), 677-683. http://dx.doi.org/ 10.1103/RevModPhys.40.677

Newman, R., \& Chrenko, R. M. (1959). Optical properties of nickel oxide. Physical Review, 114(6), 1507-1513. http://dx.doi.org/10.1103/PhysRev.114.1507

Ono, S., Komiya, S., \& Ando, Y. (2007). Strong charge fluctuations manifested in the high-temperature Hall coefficient of high-T c cuprates. Physical Review B, 75(2), 024515. http://dx.doi.org/10.1103/PhysRevB. 75.024515

Perkins, J. D., Birgeneau, R. J., Graybeal, J. M., Kastner, M. A., \& Kleinberg, D. S. (1998). Midinfrared optical excitations in undoped lamellar copper oxides. Physical Review B, 58(14), 9390-9401. http://dx.doi.org/ 10.1103/PhysRevB.58.9390 
Perkins, J. D., Graybeal, J. M., Kastner, M. A., Birgeneau, R. J., Falck, J. P., \& Greven, M. (1993). Mid-infrared optical absorption in undoped lamellar copper oxides. Physical review letters, 71(10), 1621-1624. http://dx.doi.org/10.1103/PhysRevLett.71.1621

Preyer, N. W., Birgeneau, R. J., Chen, C. Y., Gabbe, D. R., Jenssen, H. P., Kastner, M. A., ... \& Thio, T. (1989). Conductivity and Hall coefficient in $\mathrm{La} 2 \mathrm{Cu} \mathrm{O} 4+$ y near the insulator-metal transition. Physical Review B, 39(16), 11563-11569. http://dx.doi.org/10.1103/PhysRevB.39.11563

Reimers, J. R., \& Hush, N. S. (2004). Hamiltonian operators including both symmetric and antisymmetric vibrational modes for vibronic coupling and intervalence charge-transfer applications. Chemical physics, 299(1), 79-82. http://dx.doi.org/10.1016/j.chemphys.2003.12.010

Reznik, D., Pintschovius, L., Ito, M., Iikubo, S., Sato, M., Goka, H., ... \& Tranquada, J. M. (2006). Electronphonon coupling reflecting dynamic charge inhomogeneity in copper oxide superconductors. Nature, 440(7088), 1170-1173. http://dx.doi.org/10.1038/nature04704

Takagi, H., Batlogg, B., Kao, H. L., Kwo, J., Cava, R. J., Krajewski, J. J., \& Peck Jr, W. F. (1992). Systematic evolution of temperature-dependent resistivity in $\mathrm{La} 2-\mathrm{x} \mathrm{Sr} \times \mathrm{CuO} 4$. Physical review letters, 69(20), 2975. http://dx.doi.org/10.1103/PhysRevLett.69.2975

Thomas, G. A., Rapkine, D. H., Cooper, S. L., Cheong, S. W., \& Cooper, A. S. (1991). Dynamics of an excited electron in a cuprate antiferromagnet. Physical review letters, 67(20), 2906-2909. http://dx.doi.org/10.1103/ PhysRevLett.67.2906

Thomas, G. A., Rapkine, D. H., Cooper, S. L., Cheong, S. W., Cooper, A. S., Schneemeyer, L. F., \& Waszczak, J. V. (1992). Optical excitations of a few charges in cuprates. Physical Review B, 45(5), 2474-2479 http://dx.doi.org/10.1103/PhysRevB.45.2474

Timusk, T., \& Statt, B. (1999). The pseudogap in high-temperature superconductors: an experimental survey. Reports on Progress in Physics, 62(1), 61. http://dx.doi.org/10.1088/0034-4885/62/1/002

Tokura, Y., Koshihara, S., Arima, T., Takagi, H., Ishibashi, S., Ido, T., \& Uchida, S. (1990). Cu-O network dependence of optical charge-transfer gaps and spin-pair excitations in single-CuO 2-layer compounds. Physical Review B, 4l(16), 11657-11660. http://dx.doi.org/10.1103/PhysRevB.41.11657

Tranquada, J. M., Sternlieb, B. J., Axe, J. D., Nakamura, Y., \& Uchida, S. (1995). Evidence for stripe correlations of spins and holes in copper oxide superconductors. Nature, 375(6532), 561-563. http://dx.doi.org/10.1038/375561a0

Tranquada, J. M., Woo, H., Perring, T. G., Goka, H., Gu, G. D., Xu, G., ... \& Yamada, K. (2004). Quantum magnetic excitations from stripes in copper oxide superconductors. Nature, 429(6991), 534-538. http://dx.doi.org/10.1038/nature02574

Uchida, S., Ido, T., Takagi, H., Arima, T., Tokura, Y., \& Tajima, S. (1991). Optical spectra of La 2- x Sr x CuO 4: Effect of carrier doping on the electronic structure of the $\mathrm{CuO} 2$ plane. Physical Review B, 43(10), 7942-7954. http://dx.doi.org/10.1103/PhysRevB.43.7942

\section{Copyrights}

Copyright for this article is retained by the author(s), with first publication rights granted to the journal.

This is an open-access article distributed under the terms and conditions of the Creative Commons Attribution license (http://creativecommons.org/licenses/by/3.0/). 